PROCEEDINGS OF THE

AMERICAN MATHEMATICAL SOCIETY

Volume 141, Number 4, April 2013, Pages 1393-1404

S 0002-9939(2012)11665-X

Article electronically published on September 5, 2012

\title{
THE SMALLEST HAKEN HYPERBOLIC POLYHEDRA
}

\author{
CHRISTOPHER K. ATKINSON AND SHAWN RAFALSKI
}

(Communicated by Michael Wolf)

\begin{abstract}
We determine the lowest volume hyperbolic Coxeter polyhedron whose corresponding hyperbolic polyhedral 3-orbifold contains an essential 2 suborbifold, up to a canonical decomposition along essential hyperbolic triangle 2-suborbifolds.
\end{abstract}

\section{INTRODUCTION}

The organization of the volumes of hyperbolic 3-manifolds and 3-orbifolds is ongoing. Gabai, Meyerhoff and Milley have identified the Weeks-Fomenko-Matveev manifold as the lowest volume hyperbolic 3-manifold [10, 11, and Gehring, Marshall and Martin have identified the lowest volume hyperbolic 3-orbifolds [12, 15]. Restricting to the case of orbifolds, a natural class to consider, from the standpoint of volume organization, is that of polyhedral 3-orbifolds, i.e., the orientable 3 -orbifolds that correspond to tilings of hyperbolic 3 -space $\mathbb{H}^{3}$ by finite volume Coxeter polyhedra. For any hyperbolic 3-orbifold that is diffeomorphic to the interior of a compact orbifold, there is a canonical decomposition, due to Dunbar, along totally geodesic hyperbolic turnovers (which are quotients of $\mathbb{H}^{2}$ by hyperbolic triangle groups) into components that either contain an embedded, essential 2-suborbifold or contain no embedded, essential 2-suborbifolds. Moreover, because the decomposing turnovers are totally geodesic, this decomposition is volume additive, and so it is natural to consider the lowest volume hyperbolic polyhedral 3 -orbifolds (or their associated polyhedra) that either contain or do not contain essential 2-suborbifolds, up to the Dunbar decomposition. The lowest volume polyhedral orbifolds in the latter case (called small orbifolds) have been identified by Rafalski [20]. This paper addresses the former case, or what are called Haken polyhedra.

We prove the following theorem:

Theorem 1.1. The smallest volume Haken hyperbolic Coxeter polyhedron is the Lambert cube $\mathcal{C}$.

The Lambert cube is combinatorially a cube with all but three of its dihedral angles equal to $\pi / 2$. The remaining three dihedral angles equal $\pi / 3$, as in Figure 1 , The Lambert cube is an example of a hyperbolic Coxeter $n-$ prism. For each $n \geq 5$, there is a (Haken) $n$-prism that is conjectured to be the lowest volume hyperbolic polyhedron with $2 n$ vertices.

Received by the editors August 19, 2011.

2010 Mathematics Subject Classification. Primary 52B10, 57M50, 57R18.

Key words and phrases. Hyperbolic polyhedra, 3-dimensional Coxeter polyhedra, hyperbolic orbifold, polyhedral orbifold, hyperbolic volume, Haken orbifold.

(C) 2012 American Mathematical Society Reverts to public domain 28 years from publication 


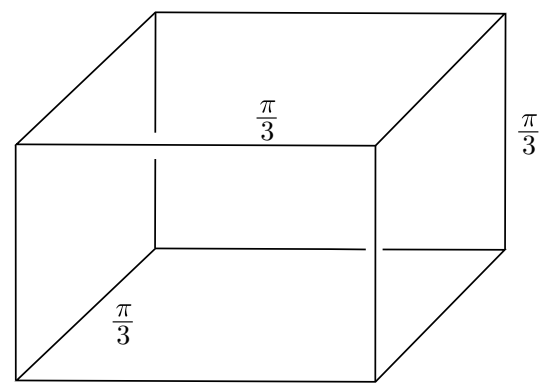

Figure 1. The smallest hyperbolic Coxeter polyhedron whose associated polyhedral 3-orbifold contains an essential 2-orbifold but contains no essential triangular 2-orbifolds. All unlabeled edges have dihedral angle $\pi / 2$.

The main idea of the proof of Theorem 1.1 is to use various volume bounds for hyperbolic polyhedra to restrict the combinatorial type of a polyhedron with volume less than the volume of the Lambert cube. The organization of the paper is as follows: In Section 2 we discuss the background on hyperbolic polyhedra and orbifolds in order to understand the main result. In Section 3 we prove a volume bound for certain types of hyperbolic polyhedra. This volume bound will be used to prove the main theorem. In Section 4, we prove Theorem 1.1

\section{BACKGROUND AND DEFINITIONS}

In this section we recall the basics of hyperbolic polyhedra and relevant definitions for orbifolds.

An abstract polyhedron is a cellulation of $S^{2}$ realizable as a convex Euclidean polyhedron. A theorem of Steinitz [22] says that realizability as a convex Euclidean polyhedron is equivalent to the 1-skeleton of the cellulation being a 3 connected planar graph (a graph is 3 -connected if the removal of any two vertices along with their incident open edges leaves the complement connected). A labeled abstract polyhedron $(P, \Theta)$ is an abstract polyhedron $P$ along with a function $\Theta: \operatorname{Edges}(P) \rightarrow(0, \pi / 2]$ labeling the edges by real numbers which should be thought of as dihedral angles. A labeled abstract polyhedron $(P, \Theta)$ is realizable as a hyperbolic polyhedron $\mathcal{P}$ if there is a label-preserving graph isomorphism between $(P, \Theta)$ and the 1 -skeleton of $\mathcal{P}$ labeled by dihedral angles.

A $k$-circuit is a simple closed curve of $k$ edges in $P^{*}$, the planar dual to $P$. A prismatic $k$-circuit is a $k$-circuit such that no two edges lie in a common face of $P^{*}$.

Andreev's theorem characterizes nonobtuse hyperbolic polyhedra in terms of combinatorial conditions on their 1-skeleta. The proof of Theorem 1.1 uses Andreev's theorem to restrict the combinatorics of certain polyhedra [3, 4].

Theorem 2.1 (Andreev's theorem). A nonobtuse labeled abstract polyhedron $(P, \Theta)$ that has more than 4 vertices is realizable as a finite volume hyperbolic polyhedron if and only if the following hold:

(1) Each vertex meets 3 or 4 edges.

(2) If $e_{i}, e_{j}$, and $e_{k}$ share a vertex, then $\Theta\left(e_{i}\right)+\Theta\left(e_{j}\right)+\Theta\left(e_{k}\right) \geq \pi$. 
(3) If $e_{i}, e_{j}, e_{k}$, and $e_{l}$ share a vertex, then $\Theta\left(e_{i}\right)+\Theta\left(e_{j}\right)+\Theta\left(e_{k}\right)+\Theta\left(e_{l}\right)=2 \pi$.

(4) If $e_{i}, e_{j}$, and $e_{k}$ form a prismatic 3-circuit, then $\Theta\left(e_{i}\right)+\Theta\left(e_{j}\right)+\Theta\left(e_{k}\right)<\pi$.

(5) If $e_{i}, e_{j}, e_{k}$, and $e_{l}$ form a prismatic 4-circuit, then $\Theta\left(e_{i}\right)+\Theta\left(e_{j}\right)+\Theta\left(e_{k}\right)+$ $\Theta\left(e_{l}\right)<2 \pi$.

(6) If $P$ has the combinatorial type of a triangular prism with edges $e_{i}, e_{j}, e_{k}$, $e_{p}, e_{q}, e_{r}$ along the triangular faces, then $\Theta\left(e_{i}\right)+\Theta\left(e_{j}\right)+\Theta\left(e_{k}\right)+\Theta\left(e_{p}\right)+$ $\Theta\left(e_{q}\right)+\Theta\left(e_{r}\right)<3 \pi$.

(7) If faces $F_{i}$ and $F_{j}$ meet along an edge $e_{i j}$, faces $F_{j}$ and $F_{k}$ meet along an edge $e_{j k}$, and $F_{i}$ and $F_{k}$ intersect in exactly one ideal vertex distinct from the endpoints of $e_{j k}$ and $e_{i j}$, then $\Theta\left(e_{i j}\right)+\Theta\left(e_{j k}\right)<\pi$.

Up to isometry, the realization of an abstract polyhedron is unique. The ideal vertices of the realization are exactly those degree 3 vertices for which there is equality in condition (2) and the degree 4 vertices.

We recall some necessary facts about orbifolds here and refer the reader to several excellent resources [7, 8. A hyperbolic Coxeter polyhedron is a hyperbolic polyhedron all of whose dihedral angles are integer submultiples of $\pi$. To any finite volume hyperbolic Coxeter polyhedron $\mathcal{P}$, there corresponds a hyperbolic 3 -orbifold $\mathcal{O}_{\mathcal{P}}$ obtained as the quotient space of $\mathbb{H}^{3}$ by the discrete group of isometries generated by all the rotations of the form $\rho \sigma$, where $\rho$ and $\sigma$ are reflections in two adjacent faces of $\mathcal{P}$. We call $\mathcal{O}_{\mathcal{P}}$ a hyperbolic polyhedral 3-orbifold. It is topologically the 3 -sphere with a marked graph that corresponds to the 1 -skeleton of $\mathcal{P}$ (a general polyhedral 3-orbifold is topologically the 3-sphere with a marked graph that corresponds to the 1-skeleton of a polyhedron that is not necessarily hyperbolic). A hyperbolic turnover is a 2-dimensional orbifold with underlying space the 2-sphere and singular locus consisting of three integer-marked points for which the sum of the reciprocals of the integral markings is less than 1 . It is a consequence of a theorem of Dunbar ([9], [7, Theorem 4.8]) that the 3-dimensional polyhedral orbifold corresponding to a hyperbolic Coxeter polyhedron can be decomposed (uniquely, up to isotopy) along a system of essential, pairwise nonparallel hyperbolic turnovers into compact, irreducible components (with turnover boundary components, if the system is nonempty) that contain no essential (embedded) turnovers, and such that each component is of one of the following types:

(1) A 3-orbifold that contains an essential 2-suborbifold (that is not a turnover), or

(2) A 3-orbifold that contains no essential 2-suborbifolds but that is not the product of a turnover with an interval, or

(3) A 3-orbifold that is the product of a hyperbolic turnover with an interval. This decomposition is equivalent to cutting the planar projection of $\partial P$ along all prismatic 3-circuits (cf. [6, Corollary 3]). We call the collection of components of types (1) and (2) the Dunbar decomposition of the hyperbolic polyhedron. Components of type (1) are called Haken, and components of type (2) are called small. A hyperbolic Coxeter polyhedron is called Haken or small if its decomposition consists of a single component of type (1) or (2), respectively.

A hyperbolic turnover in the 3 -orbifold corresponding to a hyperbolic polyhedron can either be made totally geodesic by an isotopy or else it doubly covers an embedded, nonorientable totally geodesic triangular 2-orbifold that corresponds to a triangular face, with all right dihedral angles, of the polyhedron (e.g. 16, Chapter IX.C], [1, Theorem 2.1]). Therefore, the Dunbar decomposition of a hyperbolic 
polyhedron divides the volume of the polyhedron additively, and so it is natural, in attempting to organize hyperbolic polyhedral volumes, to consider the organization up to this decomposition. The small hyperbolic Coxeter polyhedra have been classified by Rafalski [20, Theorem 1.1]:

Theorem 2.2. A 3-dimensional hyperbolic Coxeter polyhedron is small if and only if it is a generalized tetrahedron (see Figure 2).

A generalized hyperbolic tetrahedron is a hyperbolic tetrahedron for which any or all of the vertices are allowed to be ideal or the truncations of hyperideal points (see the discussion of truncated tetrahedra in Section 3). There are nine generalized tetrahedra with all four generalized vertices being finite (e.g., [21, Chapter 7]). Every other generalized tetrahedron is obtained from one (or more) of these nine by decreasing the dihedral angles of some collection of edges, a process that increases volume, by Schläfli's formula [17. Of the nine generalized tetrahedra with all finite generalized vertices, the one of lowest volume is the 3-5-3 Coxeter tetrahedron. Its volume to six decimal places is 0.039050 . Theorem 1.1 determines the lowest volume Haken hyperbolic polyhedron.

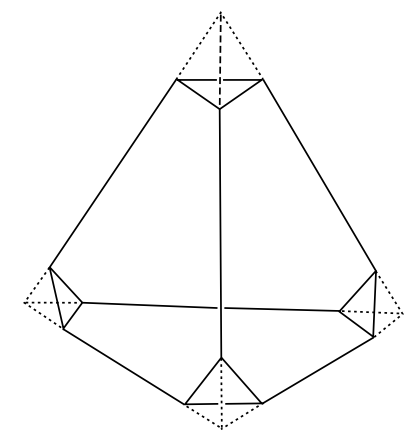

Figure 2. The small Coxeter polyhedra in $\mathbb{H}^{3}$

\section{ON POLYHEDRAL VOLUMES}

The main result of this section is Proposition [3.6, in which we improve on a result of Atkinson giving a lower bound on the volume of a hyperbolic polyhedron of graph type [6]. Theorem 1.1 also requires techniques developed by Atkinson [5, 6, which we recall here.

We begin with the following theorem that bounds the volume of a hyperbolic polyhedron without prismatic 4 -circuits in terms of the number of vertices $\underline{6}$. Theorem 1.1]. This theorem will be used in our proof of Theorem 1.1 in Section 4 to show that any polyhedron with volume less than that of the Lambert cube must contain a prismatic 4 -circuit.

Theorem 3.1. Let $\mathcal{P}$ be a nonobtuse hyperbolic polyhedron containing no prismatic 4-circuits, $N_{4}$ degree-4 vertices, and $N_{3}$ degree-3 vertices. Then

$$
\frac{4 N_{4}+N_{3}-8}{32} \cdot V_{8}<\operatorname{Vol}(\mathcal{P}),
$$

where $V_{8}=3.663862 \ldots$ is the volume of the regular ideal hyperbolic octahedron. 
The idea behind the proof of Theorem 3.1 is to deform $\mathcal{P}$ to a right-angled polyhedron through an angle-nondecreasing family of polyhedra. By Schläfli's formula, this deformation does not increase volume. The assumption that there are no prismatic 4-circuits ensures that all polyhedra in this family are hyperbolic. The lower bound then comes from applying Miyamoto's theorem, discussed below, to the resulting right-angled polyhedron 5 .

In order to prove Theorem 1.1, we will also need to show that polyhedra with certain types of prismatic 4-circuits also have volumes exceeding that of the Lambert cube. However, the techniques used in Theorem 3.1 do not work in the presence of prismatic 4-circuits, because attempting such a deformation can cause some or all of the polyhedron to degenerate to a Seifert fibered polyhedral orbifold. Although there are volume bounds for such polyhedra [6, Theorem 13], they give a lower bound of 0 for an infinite family of polyhedra of graph type. In Proposition 3.6, we improve this to give a nonzero lower bound for all hyperbolic polyhedra of graph type. A polyhedron $\mathcal{P}$ is of graph type if the polyhedron $\mathcal{P}^{\perp}$ obtained by replacing all dihedral angles by $\pi / 2$ corresponds to a graph orbifold (i.e., an orbifold with no atoroidal components in its geometric decomposition). Atkinson has shown that all such polyhedra are obtained by gluing right-angled prisms along quadrilateral faces and that the components of the geometric decomposition of the 3 -orbifold for $\mathcal{P}^{\perp}$ correspond exactly to the prisms that were glued [6, Sections 4.2 and 6.4].

The remainder of this section focuses on proving Proposition 3.6. The proof is an application of Miyamoto's theorem, which we recall below. We first describe the relevant background.

In the projective model of $\mathbb{H}^{3}$, thought of as the open ball in $\mathbb{R}^{3} \cup\{\infty\}$, consider any linearly independent set of 4 points that lie outside $\mathbb{H}^{3}$ such that the line between any two points meets $\overline{\mathbb{H}^{3}}$ in at least one point. The intersection of the convex hull of these points with $\mathbb{H}^{3}$ is an infinite volume polyhedron, provided at least one of the points lies outside $\overline{\mathbb{H}^{3}}$. Form a finite volume polyhedron by truncating the vertices that lie outside $\overline{\mathbb{H}^{3}}$ by their polar hyperplanes. The result is a truncated tetrahedron. A truncated tetrahedron is regular if every edge between a pair of truncating planes has the same length.

Define $\rho_{3}(r)$ to be the ratio of the volume of the regular truncated tetrahedron of edge length $r$ to the sum of the areas of its faces. The dihedral angle $\theta$ along an edge of a regular truncated tetrahedron is determined by the edge length $r$ via the formula

$$
\cosh r=\frac{\cos \theta}{2 \cos \theta-1}
$$

Denote the truncated tetrahedron with dihedral angle $\theta$ by $T_{\theta}$. The explicit calculation of $\rho_{3}(r)$ was given by Miyamoto [18]:

$$
\begin{aligned}
\rho_{3}(r) & =\frac{\operatorname{Vol}\left(T_{\theta(r)}\right)}{4(\pi-3 \theta(r))} \\
& =\frac{1}{4(\pi-3 \theta(r))}\left(V_{8}-3 \int_{0}^{\theta(r)} \cosh ^{-1}\left(\frac{\cos (t)}{2 \cos (t)-1}\right) d t\right),
\end{aligned}
$$

where $\theta(r)$ is defined implicitly by (3.1). Miyamoto also proved that $\rho_{3}$ is increasing in $r$ [18, Lemma 2.1]. Using (3.1), $\rho_{3}$ is also easily shown to be increasing in $\theta$. 
A return path in an orbifold with totally geodesic boundary is an orthogeodesic segment with endpoints on the geodesic boundary.

We can now state Miyamoto's theorem. Miyamoto proved this theorem for all dimensions $n \geq 2$, but we require only the 3-dimensional case.

Proposition 3.2. If a complete hyperbolic 3-orbifold $Q$ of finite volume with totally geodesic boundary has a lower bound $l \geq 0$ for the length of its return paths, then

$$
\operatorname{Vol}(Q) \geq \rho_{3}\left(\frac{l}{2}\right) \operatorname{Area}(\partial Q) .
$$

If $F_{1}, \ldots, F_{r}$ is a collection of faces of a hyperbolic polyhedron $\mathcal{P}$ such that the sides of each $F_{i}$ all have dihedral angles of $\pi / 2$ in $\mathcal{P}$ and such that no two faces share an edge, then this collection of faces corresponds to a collection of embedded, totally geodesic polygonal 2-suborbifolds with mirrored sides in the hyperbolic polyhedral 3 -orbifold $\mathcal{O}_{\mathcal{P}}$ obtained from $\mathcal{P}$. If $Q$ is the hyperbolic 3 -orbifold with totally geodesic boundary obtained by cutting $\mathcal{O}_{\mathcal{P}}$ along this collection of 2 -orbifolds and taking the metric completion, then Miyamoto's theorem applies. In particular, the inequality that gives the volume bound may be divided by 2 to give

$$
\operatorname{Vol}(\mathcal{P}) \geq \rho_{3}\left(\frac{l}{2}\right) \sum_{i=1}^{r} \operatorname{Area}\left(F_{i}\right)
$$

To get the best volume bound from Miyamoto's theorem, we use the following special case of a proposition of Rafalski [19, Proposition 8.2] to calculate the lower bound for the length of a return path in an orbifold in terms of the Euler characteristic of the boundary. A return path is closed if its endpoints are equal. This can happen, for example, if the orthogeodesic encounters an order-2 elliptic axis.

Proposition 3.3. Let $Q$ be a complete hyperbolic 3-orbifold with closed totally geodesic boundary. Then $Q$ has a shortest return path $\gamma$, and there is a positive integer $k$ such that the length of $\gamma$ is at least the edge length of $T_{\theta}$, where

$$
\theta=\frac{\pi}{3(1-k \chi(\partial Q))}
$$

and where $k>1$ if and only if $\gamma$ is contained in a singular axis of (maximal) order $k$ in $Q$ (i.e., $\gamma$ meets a cone point of order $k$ in $\partial Q$ ).

Combining Proposition 3.3 with Proposition 3.2 , we obtain the following theorem, which gives a lower bound on the volume of a hyperbolic 3-orbifold with totally geodesic boundary in terms of the Euler characteristic of the boundary.

Theorem 3.4. Suppose that $Q$ is a hyperbolic 3-orbifold with totally geodesic boundary $\partial Q$. Let

$$
f(t)=\cosh ^{-1}\left(\frac{\cos t}{2 \cos t-1}\right)
$$

Then if $k$ is the maximal order of an elliptic element of $\pi_{1}(\partial Q), x=-\chi(\partial Q)$, and

$$
R=1 / 2 \cdot f\left(\frac{\pi}{3(1+k x)}\right)
$$

we have

$$
\operatorname{Vol}(Q) \geq \frac{2 \pi x}{4\left(\pi-3 f^{-1}(R)\right)}\left(V_{8}-3 \int_{0}^{f^{-1}(R)} f(t) d t\right)
$$


Proof. Miyamoto proved that $\rho_{3}(r)$ is increasing with respect to both $r$ and $\theta$. Because the lower bound for $l$ is determined, in Proposition 3.3, as the edge length of a regular truncated tetrahedron $T_{\theta}$ (where $\theta$ depends on the Euler characteristic of the orbifold boundary), and because the edge length is minimized when $\theta$ is minimized, we consider the conditions on a return path that minimize $\theta$. Referring to the statement of Proposition 3.3 , we can conclude that $\theta$ is minimized when $k$ is largest. The conclusion of the theorem follows by using (3.1) along with Proposition 3.3 to expand the volume bound given by Proposition 3.2

To a polyhedron $\mathcal{P}$ of graph type that is not a prism, we associate a graph $G(\mathcal{P})$ with vertex set consisting of the set of prisms in the canonical geometric decomposition of $\mathcal{P}^{\perp}$ and edges between any two vertices whose corresponding geometric pieces are glued along a quadrilateral. A similar definition could be made in the case of general graph orbifolds. Note that in the case of polyhedral orbifolds, $G(\mathcal{P})$ is a tree, and so has at least two degree- 1 vertices. The number of degree1 vertices of $G(\mathcal{P})$ can be used to bound the volume of $\mathcal{P}$ below. The following lemma then shows that each degree-1 vertex of $G(\mathcal{P})$ yields a quadrilateral face that corresponds, after a volume-nonincreasing deformation of $\mathcal{P}$, to an embedded totally geodesic suborbifold in the resulting 3-orbifold.

Lemma 3.5. Suppose that $\mathcal{P}$ is a hyperbolic polyhedron of graph type (that is not a prism) such that $G(\mathcal{P})$ has $m$ degree-1 vertices. Then there exists a volumenonincreasing deformation of $\mathcal{P}$ to a hyperbolic polyhedron $\mathcal{P}^{\prime}$ for which $\mathcal{O}_{\mathcal{P}^{\prime}}$ contains a totally geodesic suborbifold of area at least $\frac{m \pi}{6}$.

Proof. We show that there exists an angle-nondecreasing deformation of $\mathcal{P}$ to a polyhedron $\mathcal{P}^{\prime}$ such that all edges of the quadrilaterals have dihedral angle $\pi / 2$, and that $\mathcal{P}^{\prime}$ satisfies Andreev's theorem.

Each degree-1 vertex $v$ corresponds to a Seifert fibered component $Q_{v}$ whose singular locus has the combinatorial type of an $n$-prism, $n \geq 5$. The fact that $n \geq 5$ follows from the fact that the vertices of $G(\mathcal{P})$ correspond to the components of the canonical geometric decomposition. Degree- 1 implies that $Q_{v}$ meets only one other component of the geometric decomposition, leaving $n-3$ quadrilateral faces free, sharing edges in a linear fashion. In each $n$-prism, choose one of these quadrilaterals.

There exists an angle-increasing deformation of a polyhedron in which each of the edges along each of the quadrilaterals has dihedral angle $\pi / 2$ [6, Lemma 12]. Then the interior angles of the quadrilaterals are equal to the dihedral angles along the edges emanating from the corresponding vertices. Since the area of the quadrilateral is the difference of $2 \pi$ and its interior angle sum, the smallest hyperbolic Coxeter quadrilateral has area $\pi / 6$.

The preceding three results can be used to prove the following:

Proposition 3.6. Suppose that $\mathcal{P}$ is a hyperbolic polyhedron of graph type and that $\mathcal{P}$ is not a prism. Let $\mathcal{C}$ be the Lambert cube. Then

$$
\operatorname{Vol}(\mathcal{P})>\operatorname{Vol}(\mathcal{C})=0.324423 \ldots
$$

Proof. Suppose that $m$ is the number of degree- 1 vertices of $G(\mathcal{P})$. We may deform $\mathcal{P}$ in a volume-nonincreasing manner to a polyhedron $\mathcal{P}^{\prime}$, where $\mathcal{P}^{\prime}$ has all dihedral angles of the form $\pi / 2$ and $\pi / 3$ [6, Lemma 12]. By Lemma 3.5, there exists a 
collection of $m$ quadrilateral faces of $\mathcal{P}^{\prime}$ all of whose edges are labeled 2 and with total area at least $\frac{m \pi}{6}$. Each quadrilateral in this collection has between one and four vertices with an interior angle equal to $\pi / 3$ (corresponding to the dihedral angle between the two faces of the polyhedron that meet the quadrilateral at each such vertex). The other vertices have interior angles of $\pi / 2$. Let $m_{i}(i \in\{1,2,3,4\})$ denote the number of quadrilateral faces with $i$ interior angles of $\pi / 3$. Then $m=$ $\sum_{i} m_{i}$.

By the discussion in the paragraph following Proposition 3.2 , we have

$$
\operatorname{Vol}\left(\mathcal{P}^{\prime}\right) \geq \frac{\pi}{6}\left(\sum_{i=1}^{4} i m_{i}\right) \rho_{3}\left(\frac{l}{2}\right),
$$

where $l \geq 0$ is a lower bound for the length of a return path in the orbifold with boundary obtained by cutting the 3 -orbifold $\mathcal{O}_{\mathcal{P}^{\prime}}$ along the collection of totally geodesic suborbifolds corresponding to the $m$ quadrilaterals of $\mathcal{P}^{\prime}$.

We recall that $\rho_{3}$ is increasing with respect to both $r$ and $\theta$ (where $r$ is the edge length of the regular truncated tetrahedron $T_{\theta}$ with dihedral angle $\theta$ ). In particular, $\rho_{3}(0)=0.291560 \ldots$ is the minimum of this function on its domain. By (3.1), $r$ and $\theta$ each increase with respect to the other. Using the minimum of $\rho_{3}$, it is easily shown that the lower bound for volume given above is greater than $\operatorname{Vol}(\mathcal{C})$ if either of $m_{4}$ or $m_{3}$ is nonzero. The bound is also larger than $\operatorname{Vol}(\mathcal{C})$ if $m_{2} \geq 2$, if $m_{2}=1$ and $m_{1}$ is nonzero, or if $m_{1} \geq 3$. Since $m \geq 2$, we are left to consider the case when $m_{1}=2$.

The minimum of $\rho_{3}$ is insufficient to give the appropriate lower bound for $\operatorname{Vol}\left(\mathcal{P}^{\prime}\right)$ in this case. Using Theorem 3.4 with $k=3$ gives a lower bound of $0.406419 \ldots$, which is larger than $\operatorname{Vol}(\mathcal{C})$, completing the proof.

\section{Proof of the Main theOREM}

The idea behind the proof of Theorem 1.1 is to use Proposition 3.6 in conjunction with techniques established by Atkinson [5, 6] and Inoue [14] to restrict the possible combinatorial types of polyhedra with small volumes. All polyhedral volumes in the proof are calculated using known formulae and the computational software Orb, developed by Heard [13].

Theorem 1.1. The smallest volume Haken hyperbolic Coxeter polyhedron is the Lambert cube $\mathcal{C}$.

Proof. Suppose that $\mathcal{P}$ is a Haken hyperbolic Coxeter polyhedron such that $\operatorname{Vol}(\mathcal{P})$ $<\operatorname{Vol}(\mathcal{C})=0.324423 \ldots$ We will show that $\mathcal{P}$ must have the combinatorial type of a prism.

First note that $\mathcal{P}$ must contain at least one prismatic 4 -circuit. If $\mathcal{P}$ contained no prismatic 4 -circuits, then $\mathcal{P}^{\perp}$ admits a structure as a compact right-angled hyperbolic polyhedron such that $\operatorname{Vol}\left(\mathcal{P}^{\perp}\right) \leq \operatorname{Vol}(\mathcal{P})$. The smallest volume compact, right-angled polyhedron is the right-angled dodecahedron [14. But the volume of this polyhedron is $4.306207 \ldots$, which is considerably larger than $\operatorname{Vol}(\mathcal{C})$.

So we suppose that $\mathcal{P}$ has at least one prismatic 4 -circuit. There are two cases to consider: either the geometric decomposition of the orbifold $\mathcal{O}_{\mathcal{P} \perp}$ for $\mathcal{P}^{\perp}$ contains at least one atoroidal component or $\mathcal{O}_{\mathcal{P} \perp}$ is a graph orbifold (i.e., $\mathcal{P}$ is of graph type). 
In this former case, suppose that $Q$ is an atoroidal component of $\mathcal{O}_{\mathcal{P}^{\perp}}$. Then $Q$ corresponds to a finite volume right-angled hyperbolic polyhedron $\mathcal{Q}$ as well as to a subset $Q_{\mathcal{P}}$ of $\mathcal{P}$ for which we have $\operatorname{Vol}(\mathcal{Q}) \leq \operatorname{Vol}\left(Q_{\mathcal{P}}\right) \leq \operatorname{Vol}(\mathcal{P})$ [6. Propositions 2 and 3]. The proof of this fact uses a result of Agol, Storm and Thurston 2, Corollary 2.2]. The polyhedron $\mathcal{Q}$ has at least one 4 -valent ideal vertex coming from the prismatic 4 -circuit. The following lemma shows that the volume of $\mathcal{Q}$ must exceed that of the Lambert cube.

Lemma 4.1. Suppose $\mathcal{Q}$ is a right-angled hyperbolic polyhedron with at least one ideal vertex. Then $\operatorname{Vol}(\mathcal{Q})>\operatorname{Vol}(\mathcal{C})$.

Proof. Let $N_{3}$ and $N_{4}$ be the number of 3 -valent and 4 -valent vertices of $\mathcal{Q}$ respectively. If $N_{4} \geq 3$, then Theorem 3.1 immediately gives the conclusion in this case because

$$
\operatorname{Vol}(\mathcal{Q}) \geq \frac{4 N_{4}-8}{32} \cdot V_{8}>0.457>\operatorname{Vol}(\mathcal{C})
$$

We now discuss the cases where $N_{4}$ is either 1 or 2 . If $E$ is the number of edges of $\mathcal{Q}$, note that $2 E=3 N_{3}+4 N_{4}$.

Suppose first that $N_{4}=1$. Then $2 E=3 N_{3}+4$, so $N_{3}$ is even. The minimal number of vertices of a polyhedron is 4 , so $N_{3} \geq 3$. The only example with $N_{4}=1$ and $N_{3}=4$ is an ideal pyramid with square base which, by Andreev's theorem, does not admit a right-angled hyperbolic realization. This discussion along with the following lemma shows that a right-angled hyperbolic polyhedron with a single ideal vertex must have more than 6 finite vertices.

Lemma 4.2. There are no right-angled hyperbolic polyhedrons with $N_{4}=1$ and $N_{3}=6$.

Proof. Suppose that $\mathcal{P}$ is such a polyhedron. Label the ideal vertex $v_{0}$, the four vertices adjacent to $v_{0}$ by $v_{1}, v_{2}, v_{3}$, and $v_{4}$, and the remaining two vertices by $v_{5}$ and $v_{6}$. By Euler characteristic considerations, $\mathcal{P}$ must contain 11 edges. Denote the set $\left\{v_{1}, v_{2}, v_{3}, v_{4}\right\}$ by $\mathcal{V}$. We first observe that $\mathcal{P}$ must contain at least one edge with both endpoints in $\mathcal{V}$. To see this, note that each vertex in $\mathcal{V}$ is trivalent, so each vertex in $\mathcal{V}$ meets two more edges in addition to the edge shared with $v_{0}$. There are only seven edges in addition to the edges containing $v_{0}$, so by the pigeonhole principle, at least two of the vertices in $\mathcal{V}$ must share an edge.

In fact, there must be two edges with both endpoints in $\mathcal{V}$. Suppose that there was only one such edge. We may assume, without loss of generality, that the edge with both endpoints in $\mathcal{V}$ meets $v_{1}$ and $v_{2}$. Vertices $v_{3}$ and $v_{4}$ must have valence 3 , so both must meet two additional edges. Bigons are not permitted, so each of $v_{3}$ and $v_{4}$ must meet edges containing $v_{5}$ and $v_{6}$ giving a configuration as in Figure 3 , up to switching $v_{5}$ and $v_{6}$. Suppose for concreteness that the embedding of the 1 -skeleton is as shown. The edge path of length four passing through $v_{0}, v_{3}, v_{6}$, and $v_{4}$ separates $v_{5}$ from $v_{1}$ and $v_{2}$. This is a contradiction to planarity because $v_{5}$ must be connected to one of $v_{1}$ and $v_{2}$ by an edge.

Finally, we observe that there cannot be 3 or 4 edges with both endpoints in $\mathcal{V}$. Having 3 such edges forces a bigon between $v_{5}$ and $v_{6}$ and 4 such edges causes the graph to be disconnected.

The three possibilities for such a polyhedron with two edges having both endpoints in $\mathcal{V}$ are shown in Figure 4. In the first two cases, the graphs are not 3-connected. The third case contradicts the fourth condition of Andreev's theorem. 


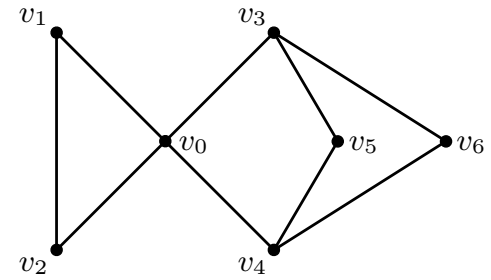

FIGURE 3
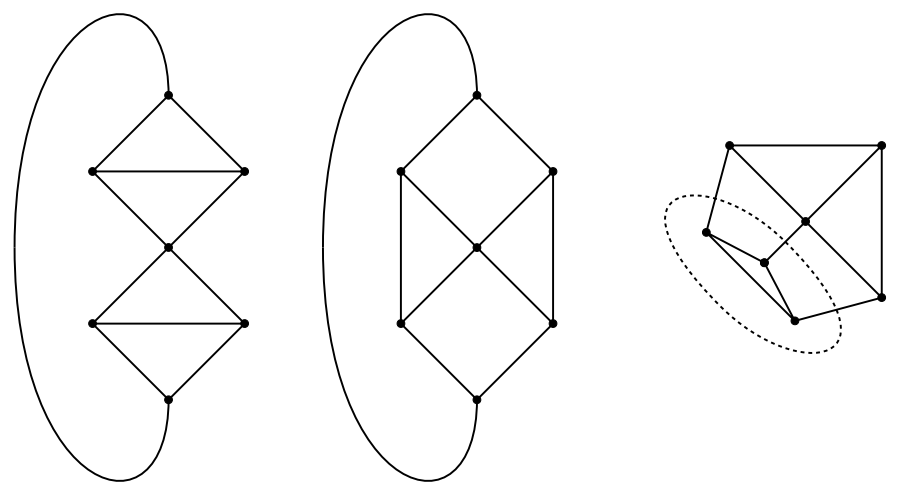

Figure 4. The first two graphs are not 3-connected. The dashed curve in the third graph illustrates the contradiction to Andreev's theorem.

It follows from Lemma 4.2 and the discussion preceding it that a right-angled hyperbolic polyhedron with a single ideal vertex must have at least 8 finite vertices. Theorem 3.1 then says

$$
\operatorname{Vol}(\mathcal{Q})>\frac{4 \cdot 1+8-8}{32} \cdot V_{8}>0.458>\operatorname{Vol}(\mathcal{C})
$$

If $N_{4}=2$, then $2 E=3 N_{3}+8$, so $N_{3}$ is odd. Then since $N_{3} \geq 3$, Theorem 3.1 gives

$$
\operatorname{Vol}(\mathcal{Q})>\frac{4 \cdot 2+3-8}{32} \cdot V_{8}>0.343>\operatorname{Vol}(\mathcal{C})
$$

This completes the proof of Lemma 4.1 .

To complete the proof of Theorem 1.1, we are left to consider the case when $\mathcal{P}$ is a polyhedron of graph type. If $\mathcal{P}$ is not a prism, then Proposition 3.6 implies that the volume of $\mathcal{P}$ exceeds that of $\mathcal{C}$. Hence if $\operatorname{Vol}(\mathcal{P}) \leq \operatorname{Vol}(\mathcal{C}), \mathcal{P}$ must have the combinatorial type of a prism.

Any hyperbolic Coxeter 3-prism (i.e., a triangular prism) is either a generalized tetrahedron with one truncated vertex or has a Dunbar decomposition into two generalized tetrahedra each with one truncated vertex [20, Lemma 3.1]. In either case, such a prism is not Haken. Atkinson has determined the smallest volume Coxeter $n$-prisms for $n \geq 5$ and has shown that the smallest volumes increase monotonically in $n$ [6, Theorem 11 and Corollary 8]. The lowest 5 -prism volume is $0.763304 \ldots$, greater than that of $\mathcal{C}$. The 4 -prism case remains; that is, we must show that $\mathcal{C}$ has the smallest volume among Coxeter polyhedra with the combinatorial type of the cube. 

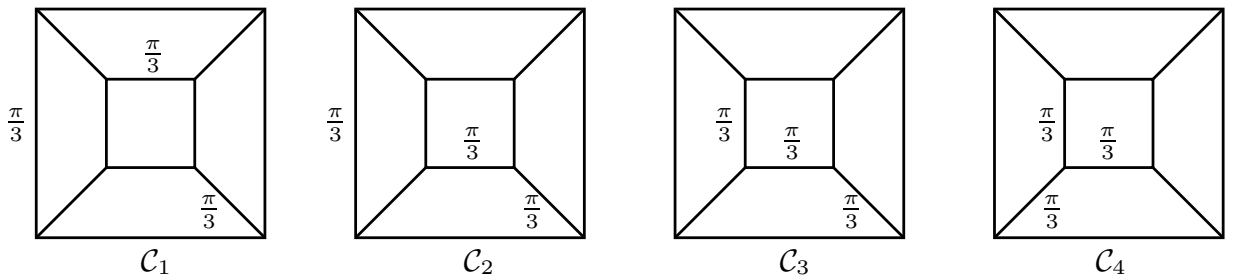

Figure 5. Lowest volume candidates for combinatorial cubes

For any Coxeter polyhedron with the combinatorial type of the cube, there exists a volume-nonincreasing deformation to one with all dihedral angles $\pi / 2$ and $\pi / 3$ [6. Proposition 4]. The only restriction that Andreev's theorem places on such a polyhedron is that there is at least one dihedral angle of $\pi / 3$ along each of the three prismatic 4 -circuits. If there were more than one dihedral angle of $\pi / 3$ along one of the prismatic 4 -circuits, there would exist a further volume-decreasing deformation to a polyhedron with only one dihedral angle of $\pi / 3$. Hence any Coxeter polyhedron with the combinatorial type of the cube has volume at least that of a cube with a single dihedral angle of $\pi / 3$ occurring on each of the prismatic 4 -circuits and all other dihedral angles $\pi / 2$. Up to isometry, there are 4 such polyhedra: $\mathcal{C}_{1}=\mathcal{C}, \mathcal{C}_{2}$, $\mathcal{C}_{3}$, and $\mathcal{C}_{4}$, as shown in Figure 5. The respective volumes of $\mathcal{C}_{1}, \mathcal{C}_{2}, \mathcal{C}_{3}$, and $\mathcal{C}_{4}$, are $0.324423 \ldots, 0.392365 \ldots, 0.464467 \ldots$, and $0.634337 \ldots$

This completes the proof.

\section{ACKNOWLEDGMENT}

The authors wish to thank Dave Futer for helpful feedback.

\section{REFERENCES}

[1] Colin Adams and Eric Schoenfeld, Totally geodesic Seifert surfaces in hyperbolic knot and link complements. I, Geom. Dedicata 116 (2005), 237-247. MR2195448 (2006j:57008)

[2] Ian Agol, Peter A. Storm, and William Thurston, Lower bounds on volumes of hyperbolic Haken 3-manifolds, J. Amer. Math. Soc. 20 (2007), no. 4, 1053-1077. MR2328715 (2008i:53086)

[3] E. M. Andreev, On convex polyhedra in Lobachevski spaces, Math. USSR Sbornik 10 (1970), no. 3, 413-440. MR0259734 (41:4367)

[4] _ On convex polyhedra of finite volume in Lobachevski space, Math. USSR Sbornik 12 (1970), no. 2, 255-259. MR0273510(42:8388)

[5] Christopher K. Atkinson, Volume estimates for equiangular hyperbolic Coxeter polyhedra, Algebr. Geom. Topol. 9 (2009), no. 2, 1225-1254. MR2519588(2010k:57035)

[6] _ Two-sided combinatorial volume bounds for non-obtuse hyperbolic polyhedra, Geom. Dedicata 153 (2011), 177-211. MR2819670

[7] Michel Boileau, Sylvain Maillot, and Joan Porti, Three-dimensional orbifolds and their geometric structures, Panoramas et Synthèses [Panoramas and Syntheses], vol. 15, Société Mathématique de France, Paris, 2003. MR2060653 (2005b:57030)

[8] Daryl Cooper, Craig D. Hodgson, and Steven P. Kerckhoff, Three-dimensional orbifolds and cone-manifolds, MSJ Memoirs, vol. 5, Mathematical Society of Japan, Tokyo, 2000, With a postface by Sadayoshi Kojima. MR.1778789 (2002c:57027)

[9] William D. Dunbar, Hierarchies for 3-orbifolds, Topology Appl. 29 (1988), no. 3, 267-283. MR953958 (89h:57008)

[10] David Gabai, Robert Meyerhoff, and Peter Milley, Minimum volume cusped hyperbolic threemanifolds, J. Amer. Math. Soc. 22 (2009), no. 4, 1157-1215. MR2525782(2011a:57031)

[11] Mom technology and volumes of hyperbolic 3-manifolds, Comment. Math. Helv. 86 (2011), no. 1, 145-188. MR2745279 
[12] Frederick W. Gehring and Gaven J. Martin, Minimal co-volume hyperbolic lattices. I. The spherical points of a Kleinian group, Ann. of Math. (2) 170 (2009), no. 1, 123-161. MR2521113(2010h:57029)

[13] D. Heard, Orb, www.ms.unimelb.edu.au/ snap/orb.html.

[14] Taiyo Inoue, Organizing volumes of right-angled hyperbolic polyhedra, Algebr. Geom. Topol. 8 (2008), no. 3, 1523-1565. MR2443253 (2009k:57025)

[15] T. H. Marshall and Gaven J. Martin, Minimal co-volume hyperbolic lattices. II. Simple torsion in Kleinian groups (2008), Preprint.

[16] Bernard Maskit, Kleinian groups, Grundlehren der Mathematischen Wissenschaften [Fundamental Principles of Mathematical Sciences], vol. 287, Springer-Verlag, Berlin, 1988. MR959135 (90a:30132)

[17] John Milnor, The Schläfli differential formula, Collected papers. Vol. 1, Geometry, Publish or Perish Inc., 1994. MR1277810 (95c:01043)

[18] Yosuke Miyamoto, Volumes of hyperbolic manifolds with geodesic boundary, Topology 33 (1994), no. 4, 613-629. MR.1293303 (95h:57014)

[19] Shawn Rafalski, Immersed turnovers in hyperbolic 3-orbifolds, Groups Geom. Dyn. 4 (2010), no. 2, 333-376. MR2595095(2011a:57036)

[20] S Small hyperbolic polyhedra, Pacific J. Math. 255 (2011), no. 1, 191-240.

[21] John G. Ratcliffe, Foundations of hyperbolic manifolds, Graduate Texts in Mathematics, vol. 149, Springer-Verlag, New York, 1994. MR1299730 (95j:57011)

[22] E. Steinitz, Polyeder und Raumeinteilungen, Enzylk. Math. Wiss. 3 (1922), 1-139.

Department of Mathematics, Temple University, Philadelphia, Pennsylvania 19106

E-mail address: ckatkin@temple.edu

Department of Mathematics and Computer Science, Fairfield University, Fairfield, Connecticut 06824

E-mail address: srafalski@fairfield.edu 